\title{
PRELIMINARY GAOFEN-3 INSAR DEM ACCURACY ANALYSIS
}

\author{
Qianfu CHEN, Tao LI, Xinming TANG, Xiaoming GAO, Xiang ZHANG
}

Satellite Surveying and Mapping Application Center, National Administration of Surveying, Mapping and Geoinformation, Beijing, P.R. China - (chenqf, lit, tangxm, gaoxm, zhangx)@sasmac.cn

\section{Commission III, WG III/3}

KEY WORDS: GF-3 satellite, error analysis, calibration, digital elevation model, precision validation

\begin{abstract}
:
GF-3 satellite, the first C band and full-polarization SAR satellite of China with spatial resolution of 1m, was successfully launched in August 2016. We analyze the error sources of GF-3 satellite in this paper, and provide the interferometric calibration model based on range function, Doppler shift equation and interferometric phase function, and interferometric parameters calibrated using the three-dimensional coordinates of ground control points. Then, we conduct the experimental two pairs of images in fine stripmap I mode covering Songshan of Henan Province and Tangshan of Hebei Province, respectively. The DEM data are assessed using SRTM DEM, ICESat-GLAS points, and ground control points database obtained using ZY-3 satellite to validate the accuracy of DEM elevation. The experimental results show that the accuracy of DEM extracted from GF-3 satellite SAR data can meet the requirements of topographic mapping in mountain and alpine regions at the scale of 1:50000 in China. Besides, it proves that GF-3 satellite has the potential of interferometry.
\end{abstract}

\section{INTRODUCTION}

The GF-3 satellite is the unique civilian satellite for microwave imaging in the National High-Resolution Earth Observation System Major Project in China, and also the first C band and full-polarization synthetic aperture radar (SAR) satellite. GF-3 has the characteristics of high resolution imaging, large imaging width, high radiation precision, multi imaging modes. It can monitor the global sea and land information full-day and full-weather, with functions of providing both detailed and roughly observation. And by using left and right attitude maneuver, it can expand the observation range and reduce the time for response, in order to meet the needs of users in the marine, disaster management, water conservation, and meteorology industries. The GF-3 satellite has 12 imaging modes, which is the most of the existing SAR satellites, and the spatial resolution of GF-3 ranges from $1 \mathrm{~m}$ to $500 \mathrm{~m}$, while its width ranges from $10 \mathrm{~km}$ to $650 \mathrm{~km}$. Specific parameters are shown in Table 1 (Zhang, 2017). Also, by obtaining the repeat-pass SAR data by GF-3 satellite and making adjustment and orbit control of in-orbit calibration, users can fully mine the interferometry ability of SAR data to provide better service to the land, surveying and mapping industry for terrain mapping, deformation monitoring and other fields.

In order to support the experimental analysis, we apply a batch of GF-3 satellite SAR data to China Centre For Resources Satellite Data and Application (CRESDA). After screening and sorting it for the application, we select the repeat-pass SAR data with- potential interferometry for analysis, data processing and accuracy evaluation. In order to analyze and verify the interferometric results under complex terrain conditions, we choose data from mountain or alpine areas, respectively, which are located in Songshan, Henan province and Tangshan, Hebei province in China. Then, we build the model for measuring the height of InSAR (Jordan, 1980; Goldstein, 1988; Massonnet, 1993), and research the orthogonal decomposition of visual-

\begin{tabular}{|c|c|c|c|c|}
\hline NO. & Work Modes & $\begin{array}{c}\text { Incidence } \\
\text { Angle }\left(^{\circ}\right) \\
\end{array}$ & $\begin{array}{c}\text { Nominal } \\
\text { Resolution(m) }\end{array}$ & $\begin{array}{c}\text { Nominal Imaging } \\
\text { Bandwidth(km) }\end{array}$ \\
\hline 1 & spotlight(SL) & $20-50$ & 1 & $10 \times 10$ \\
\hline 2 & ultra-fine strip(UFS) & $20-50$ & 3 & 30 \\
\hline 3 & fine strip I(FSI) & $19-50$ & 5 & 50 \\
\hline 4 & fine strip II(FSII) & $19-50$ & 10 & 100 \\
\hline 5 & standard strip(SS) & $17-50$ & 25 & 130 \\
\hline 6 & narrow scan(NSC) & $17-50$ & 50 & 300 \\
\hline 7 & wide scan(WSC) & $17-50$ & 100 & 500 \\
\hline 8 & global scan(GLO) & $17-53$ & 500 & 650 \\
\hline 9 & full polarized strip I(QPSI) & $20-41$ & 8 & 30 \\
\hline 10 & full polarized strip II(QPSI) & $20-38$ & 25 & 40 \\
\hline 11 & wave imaging(WAV) & $20-41$ & 10 & $5 \times 5$ \\
\hline \multirow{2}{*}{12} & low & $10-20$ & 25 & 130 \\
\hline & extended(EXT) & $50-60$ & 25 & 80 \\
\hline
\end{tabular}

Table 1. The main technical specifications of each imaging mode 
vector model and three-dimensional reconstruction model (Wang, 2011a; Hua, 2014; Wang, 2003b). Interferometric error sources are analyzed (Chen, 2016a; Chen, 2016b), then we use ground control points (GCPs) for the interferometric calibration model optimization (Gonzalez, 2010; Wu, 2017) and generate related DEM products (Gao, 2017). Then we use data of SRTM DEM, the data of Geoscience Laser Altimeter System (GLAS) in Ice Cloud and Land Elevation Satellite (ICESat) (Zwally, 2002), as well as ground control points database of ZY-3 satellite (GCPD-ZY-3) to evaluate the accuracy of elevation (Krieger, 2012). The experimental results show that the accuracy of DEM extracted with GF-3 satellite SAR data using InSAR processing can meet the requirements of topographic mapping in mountain and alpine regions at the scale of 1:50000 in China. Meanwhile, we prove that GF-3 satellite has the interferometric potential, which can be further excavated for more applications.

\section{INSAR HEIGHT MEASUREMENT MODEL}

The process of obtaining the three-dimensional coordinates of the ground target points by using range function, Doppler shift equation and interferometric phase function to describe the relationship between the basic measurement value of InSAR and the position of the ground target is expressed as three-dimensional reconstruction of terrain. This process can be expressed as follows.

$$
\begin{gathered}
\vec{P}=\vec{S}_{1}+\vec{R}_{1} \\
f_{\text {dop }}=-\frac{2 \vec{v} \cdot\left(\vec{S}_{1}-\vec{P}\right)}{\lambda \cdot R_{1}} \\
\varphi=\varphi_{1}+2 k \pi=\frac{2 Q \pi\left(R_{1}-R_{2}\right)}{\lambda}
\end{gathered}
$$

where $\vec{S}_{1}$ is the vector of master antenna phase center location, $\vec{P}$ is the target point three-dimensional location vector in geodetic coordinate system, $R_{1}$ is the slant range between master antenna phase center and target point, $R_{2}$ is the slant range between slave antenna phase center and target point, $f_{\text {dop }}$ is Doppler centroid frequency, $\vec{v}$ is the velocity of satellite, $\lambda$ is wavelength, $\varphi$ is absolute interferometric phase, $\varphi_{1}$ is wrapped interferometric phase, $k$ is the number of integer phase ambiguity, $Q$ is working mode, and $Q=1$ for dual-antenna mode, $Q=2$ for repeat-pass mode.

According to the orthogonal decomposition of look vector model, we decompose $\vec{R}_{1}$ into the VPQ coordinate system, and obtain the vector $\hat{r}_{v p q}$. Then, we convert the VPQ coordinate system to geocentric coordinate system using the transfer matrix $R_{v p q}^{-1}$. Where $\hat{v} \hat{p} \hat{q}$ is the orthogonal basis of unit coordinate system, and the unit vector $\hat{v}$ is defined as the flight velocity direction, $\hat{q}$ is unit vector of cross product of velocity and baseline, and $\hat{p}$ is unit vector based on the right-hand rule.
According to the orthogonal decomposition of look vector model and the geometry of InSAR under geocentric coordinate system, the target's three-dimensional location vector in geodetic coordinate system can be expressed as follows.

$$
\begin{gathered}
\vec{P}=S_{1}+R_{1} \cdot R_{v p q}^{-1} \cdot \hat{r}_{v p q} \\
\hat{r}_{v p q}=\left[\begin{array}{lll}
r_{v} & r_{p} & r_{q}
\end{array}\right]^{T} \\
R_{v p q}^{-1}=\left[\begin{array}{lll}
\frac{\vec{v}}{v} & \frac{(\vec{v} \otimes \vec{b}) \otimes \vec{v}}{|(\vec{v} \otimes \vec{b}) \otimes \vec{v}|} & \frac{(\vec{v} \otimes \vec{b})}{|(\vec{v} \otimes \vec{b})|}
\end{array}\right]
\end{gathered}
$$

where $\hat{r}_{v p q}=\left[\begin{array}{lll}r_{v} & r_{p} & r_{q}\end{array}\right]^{T}$ is the unit look vector,

$$
\begin{gathered}
r_{v}=\frac{\lambda f_{\text {dop }}}{2 v} \\
r_{p}=\frac{R_{1}}{2 b_{p v}}\left[1+\frac{b^{2}}{R_{1}^{2}}-\left(1-\frac{\Delta r}{R_{1}}\right)^{2}\right]-\frac{b_{v} r_{v}}{b_{p v}} \\
r_{q}= \pm \sqrt{1-r_{v}^{2}-r_{p}^{2}}
\end{gathered}
$$

$R_{v p q}^{-1}$ is the transfer matrix, $b_{p v}$ is the length of perpendicular baseline, $b_{v}$ is the length of parallel baseline.

From equations $4 \sim 6$ we can find that, there are several parameters such as wave length, the vector of master antenna phase center location and velocity, slant range, baseline length, phase and Doppler center frequency, which are main factors affecting the geolocation and height measurement. And some of them such as the vector of master antenna phase center location and velocity, slant range and Doppler center frequency affect the accuracy of geolocation, which can be eliminated by geometric calibration. The other error sources are related to the height measurement, such as phase and baseline length, which is the focus of this paper. Phase error is an important error source in InSAR processing, and the phase error of 1 radian can cause a height error of about $10 \mathrm{~m}$, and the millimeter level baseline measurement error can cause the elevation error of the meter level (Gonzalez, 2010; Wu, 2017; Wang, 2014c). So, it need to be considered and eliminated in the work. Therefore, the main source of error in the interferometric height measurement is the phase and baseline. In order to obtain the precise parameters, we conduct interferometric calibration using GCPs.

\section{INTERFEROMETRIC CALIBRATION MODEL}

Through above analysis, we conduct interferometric calibration using GCPs based on the three-dimensional reconstruction model under the premise of ensuring the geometric accuracy (Ding, 2017). Before establishing the interferometric calibration model, we describe the TCN coordinate system, which can be used to decompose the baseline vector. The original point $\mathrm{O}$ is the master antenna phase center location, and the axis $\mathrm{N}$ is unit vector of connection of the master 
antenna phase center to the center of the ellipsoid, the axis $\mathrm{C}$ is unit vector of cross product of axis $\mathrm{N}$ and velocity, and axis $\mathrm{T}$ is unit vector based on the right-hand rule. As we know, the component of baseline error of the along-track can be removed by registration, so we neglected this for the topographic mapping (Wu,2017). Finally, we determine interferometric parameters in this paper, which are the baseline initial length $b_{c 0}$, baseline rate $v_{c 0}$ of the axis $\mathrm{C}$, the baseline initial length $b_{n 0}$, baseline rate $v_{n 0}$ of the axis $\mathrm{N}$, and interferometric phase $\varphi$.

The corresponding error equations can be expressed as follows.

$$
\left\{\begin{array}{l}
v_{x i}=\frac{\partial F_{x i}}{\partial \varphi} \Delta \varphi+\frac{\partial F_{x i}}{\partial b_{c 0}} \Delta b_{c 0}+\frac{\partial F_{x i}}{\partial v_{c 0}} \Delta v_{c 0}+\frac{\partial F_{x i}}{\partial b_{n 0}} \Delta b_{n 0}+\frac{\partial F_{x i}}{\partial v_{n 0}} \Delta v_{n 0}-l_{x i} \\
v_{y i}=\frac{\partial F_{y i}}{\partial \varphi} \Delta \varphi+\frac{\partial F_{y i}}{\partial b_{c 0}} \Delta b_{c 0}+\frac{\partial F_{y i}}{\partial v_{c 0}} \Delta v_{c 0}+\frac{\partial F_{y i}}{\partial b_{n 0}} \Delta b_{n 0}+\frac{\partial F_{y i}}{\partial v_{n 0}} \Delta v_{n 0}-l_{y i}(10) \\
v_{z i}=\frac{\partial F_{z i}}{\partial \varphi} \Delta \varphi+\frac{\partial F_{z i}}{\partial b_{c 0}} \Delta b_{c 0}+\frac{\partial F_{z i}}{\partial v_{c 0}} \Delta v_{c 0}+\frac{\partial F_{z i}}{\partial b_{n 0}} \Delta b_{n 0}+\frac{\partial F_{z i}}{\partial v_{n 0}} \Delta v_{n 0}-l_{z i}
\end{array}\right.
$$

According to the least square theory, the correction value of the interferometric parameters is solved. The simplified expression can be expressed as follows.

$$
\Delta X=\left(A^{T} A\right)^{-1} A^{T} l
$$

where $\Delta X$ is the vector of interferometric parameters, $A$ is the matrix of coefficients of interferometric parameters, $l$ is the residual vector, and they were obtained from equation 10 .

When the root mean square error (RMSE) of the two coordinates difference of GCPs is less than the specified threshold, the iteration is stopped, then we output the corresponding adjustment results, the equation can be expressed as follows.

$$
\sqrt{\frac{1}{n} \sum_{i=1}^{n}\left|P_{i}^{m}-P_{i}^{m-1}\right|^{2}} \leq \Delta \varepsilon
$$

where $\mathrm{n}$ is the number of GCPs, $\mathrm{m}$ is the number of iterations, $P_{i}^{m}$ represents the three-dimensional coordinates, $\mathrm{i}$ is the number of GCPs, and the threshold of $\Delta \varepsilon$ can be set to $0.5 \mathrm{~m}$ or smaller.

\section{EXPERIMENTS AND ANALYSIS}

In order to conduct the experimental analysis, we apply a batch of GF-3 satellite SAR data to China Centre For Resources Satellite Data and Application (CRESDA). After screening potential interferometric data through incidence angle, perpendicular baseline, orbit parameters and other conditions, we select two of the potential interferometric data for InSAR data processing and elevation verification in this paper. The two pairs of interferometric data are located in Songshan, Henan province and Tangshan, Hebei province. Relevant parameters are shown in Table 2.

\begin{tabular}{lcc}
\hline \multicolumn{1}{c}{ Information } & Songshan & Tangshan \\
\hline master image date & 20161129 & 20170217 \\
slave image date & 20161228 & 20170318 \\
$\begin{array}{l}\text { Imaging mode } \\
\text { baseline }\end{array}$ & FSI & FSI \\
$\begin{array}{l}\text { perpendicular } \\
\text { area coverage }\end{array}$ & $858.52 \mathrm{~m}$ & $255.81 \mathrm{~m}$ \\
terrain & $\begin{array}{l}54 * 27 \mathrm{~km}^{2} \\
\text { mountain }\end{array}$ & $\begin{array}{c}55 * 39 \mathrm{~km}^{2} \\
\text { high mountain }\end{array}$ \\
\hline
\end{tabular}

Table 2. The information of the experimental data

\subsection{InSAR Processing}

The interferometric data processing steps are as follows.

We co-registrate the image pair by using intensity correlation based polynomial method, and obtain the offset polynomial. And then resample the slave image based on the offset polynomial and cutoff the invalid area for data processing. Then the interferogram map is generated by the conjugate multiplication of the master and slave image with 2 by 2 looks. The interferogram map and its partial enlarged map are shown in Figure 3-(a)/(b) and Figure 4-(a) /(b).

In order to remove the fringes caused by smooth curved earth, we simulate the terrain phase using the SRTM DEM, then subtract the phase of the terrain from the phase of original interferogram. The flattened interferogram map is shown in Figure 3-(c) and Figure 4-(c), and the coherence map can be shown in Figure 3-(d) and Figure 4-(d).

Phase is filtered using adaptive filtering method, then we choose Minimum Cost Flow (MCF) method for phase unwrapping. Using GCPs for the interferometric calibration model optimization and generate related DEM.

From Figure 3 and 4, we can find that,

(1) From Figure 3-(a) / (b) and Figure 4-(a) / (b), except the loss of coherence in some mountainous areas, both of the images have clear interferometric fringe, showing the possibility of InSAR processing.

(2) From Figure 3-(c) and Figure 4-(c), we can see that the resided fringes still remained clearly because the satellite orbit states we obtained are real-time and not precise enough. As it has no influence on the height, we ignore this.
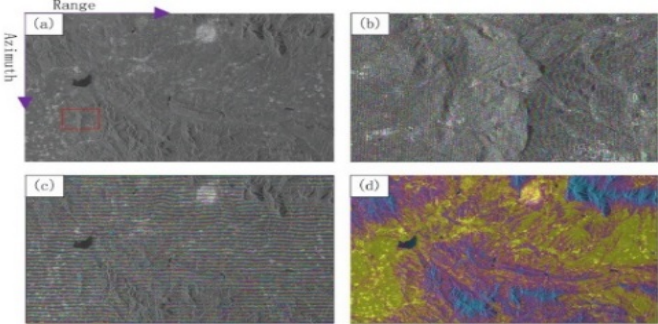

Figure 3. Interferometric processing results of Songshan. (a) is

the interferogram map; (b) is a local large map of the red rectangular box in figure (a); (c) is the flattened interferogram map; (d) is the coherence map. 


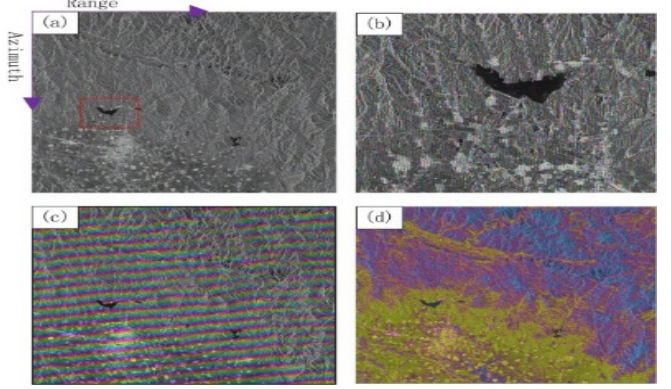

Figure 4. Interferometric processing results of Tangshan. (a) is the interferogram map; (b) is a local large map of the red rectangular box in figure (a); (c) is the flattened interferogram map; (d) is the coherence map.
(3) In Figure 3-(d) and Figure 4-(d), yellow information indicates the better coherence, and blue information indicates the worse coherence. Statistics of its coherence is shown in Figure 3, in which the ones with the coherence coefficient greater than 0.3 account for $97 \%$, while others with coherence coefficient greater than 0.7 account for $84.4 \%$. In Figure 4, the ones with the coherence coefficient greater than 0.3 and 0.47 account for $95.1 \%$ and $83.1 \%$, respectively. We can conclude that the coherence of most points in the interferogram map is good, and it is beneficial to phase unwrapping.

After phase unwrapping, the interferometric parameters are obtained by using the aforementioned algorithms, and finally we get the DEM products. The related DEMs of Songshan and Tangshan are shown in Figure 5 and Figure 6, respectively.

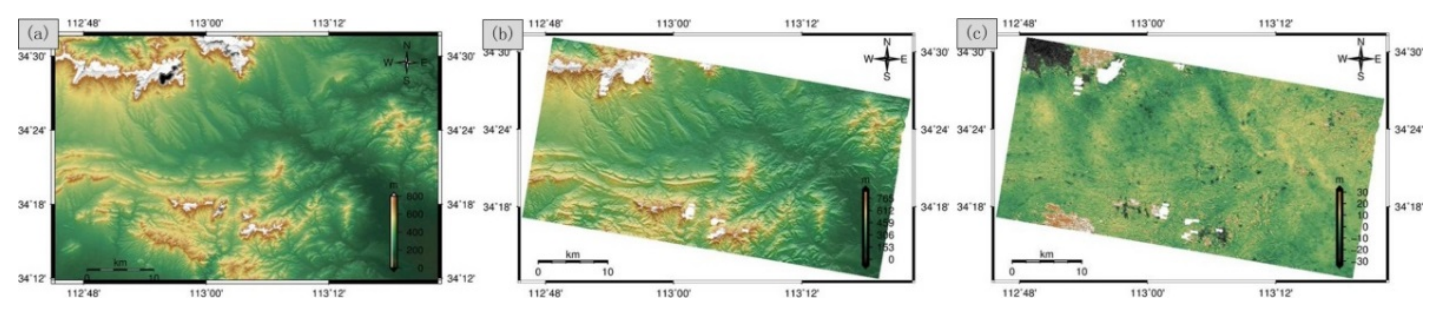

Figure 5. DEM products of Songshan region. (a) is the SRTM DEM map; (b) is the DEM generated by this paper; (c) is the elevation difference map of SRTM DEM and DEM generated by this paper.
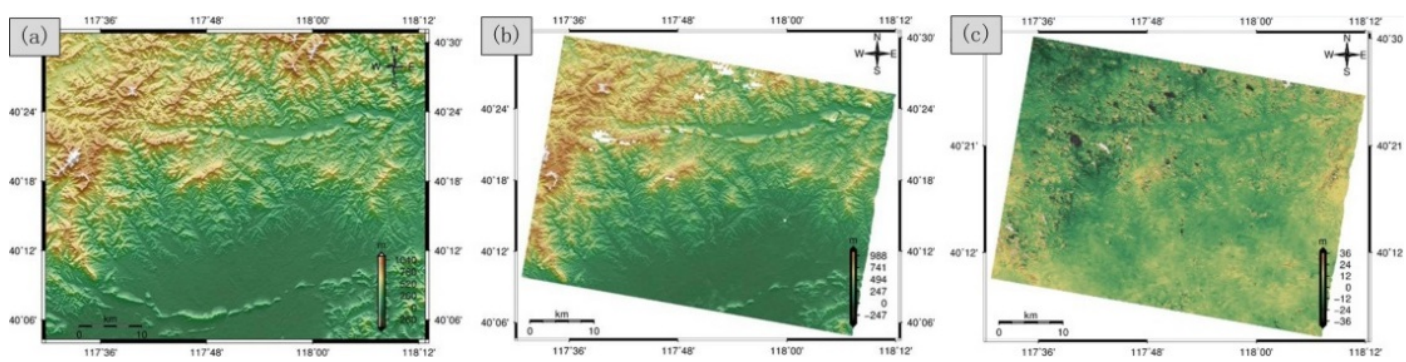

Figure 6. DEM products of Tangshan region. (a) is the SRTM DEM map; (b) is the DEM generated by this paper; (c) is the elevation difference map of SRTM DEM and DEM generated by this paper.

\subsection{Accuracy Verification of DEM}

We use SRTM DEM, ICESat-GLAS points, and ground control points database of ZY-3 satellite to validate the accuracy of DEM elevation. Its statistical results are shown in Table 7.

The following conclusions can be shown,

(1) Using the DEM obtained in this paper and SRTM DEM to do the elevation difference processing, and calculate the RMSE according to $90 \%$ point-to-point precision, we get RMSE of DEMs of Songshan and Tangshan as $5.3 \mathrm{~m}$ and $7.5 \mathrm{~m}$, respectively.

(2) Using the public ICESat-GLAS points to evaluate the accuracy of DEMs. As we know, there are a lot of invalid points among GLAS, the RMSE of SRTM DEM is $16 \mathrm{~m}$ (Farr, 2007), so we regard those points greater than 2 times RMSE as

\begin{tabular}{ccc}
\hline Reference Data & $\begin{array}{c}\text { The RMSE of } \\
\text { DEM in } \\
\text { Songshan /m }\end{array}$ & $\begin{array}{c}\text { The RMSE of } \\
\text { DEM in } \\
\text { Tangshan /m }\end{array}$ \\
\hline SRTM DEM & 5.3 & 7.5 \\
ICESat-GLAS & $6.4(2,426$ & $11.6(3,342$ \\
GCPD-ZY-3 & points $)$ & points $)$ \\
\hline
\end{tabular}

Table 7. The accuracy of DEM elevation

invalid points. Further, we keep the points of the GLAS and SRTM DEM elevation difference for less than $32 \mathrm{~m}$. Then we calculate the RMSE of GLAS points according to $90 \%$ point-to-point precision. At last, the RMSE is $6.4 \mathrm{~m}$ and $11.6 \mathrm{~m}$ in Songshan and Tangshan region, by 2,426 points and 3,342 points evaluations, respectively. 
(3) Using ground control points database of ZY-3 satellite to evaluate the accuracy of DEMs. At first, they need to be unified to the high datum of the geodetic because of inconsistency of elevation datum. And RMSE is $6.7 \mathrm{~m}$ and $8.8 \mathrm{~m}$ in Songshan and Tangshan region, respectively.

(4) Phase noise caused by low coherence, or unwrapping error of phase discontinuous region, may result in a partial elevation of information error. But these error factors are not processed or manually edited in this paper.

\section{CONCLUSION}

GF-3 satellite was successfully used in acquisition of high resolution, multi-polarization microwave images for land, surveying and mapping industry. This paper reports the interferometric ability and coherence of GF-3 satellite, then determine the main error sources, and build the interferometric calibration model and solve the parameters using GCPs. The experimental results show that the accuracy of DEM extracted with GF-3 satellite SAR data can meet the requirements of topographic mapping in mountain and alpine regions at the scale of 1:50000 in China. It proves the correctness and effectiveness of the method proposed in this paper. In addition, we will analyze the specific reasons for the loss of coherence and orbital error.

\section{ACKNOWLEDGEMENT}

The authors thank the support from America National Aeronautics and Space Administration (NASA), China Academy of Space Technology (CAST), and China Centre For Resources Satellite Data and Application (CRESDA). And this work was supported by National Key R\&D Programme of China (NO: 2017YFB0502700), Civilian Space Programme of China (DO: D010102), National Basic Surveying and Mapping Science and Technology Plan (NO: 2018KJ0204/2018KJ0304).

\section{REFERENCES}

Chen, Q., Li, T., Gao, X., Chen, W., and Wu, D., 2016. BLOCK ADJUSTMENT WITH AIRBORNE INSAR FOR HIGH-PRECISION. Proc. of IGARSS2016, pp. 6472-6475.

Chen Qianfu, Huang Guoman, Yang Shucheng, Hua Fenfen, Lu Lijun, 2017. A Method for Block Adjustment with Airborne InSAR by Aid of Three Dimensional Reconstruction Model.
Geomatics and Information Science of Wuhan University, 42(2), pp.157-162.

Ding Chibiao, Liu Jiayin, Lei Bin, et al., 2017. Preliminary exploration of systematic geolocation accuracy of GF-3 satellite system. Journal of Radars, 6(1), pp.11-16.

Farr, T. G., et al., 2007. THE SHUTTLE RADAR TOPOGRAPHY MISSION. Rev. Geophys.,45.

Gao, X., Liu, Y., Li, T., and Wu, D., 2017. High Precision DEM Generation Algorithm Based on InSAR Multi-Look Iteration. Remote Sens., 9,194.

Goldstein, R. M., Zebker, H. A., Werner, C. L., 1988. Satellite radar interferometry: Two-dimensional phase unwrapping. Radio Science,23(4), pp. 713-720.

Gonzalez, J. M., Bachmann, M., Krieger, G., and Fiedler, H., 2010. Development of the TanDEM-X Calibration Concept: Analysis of Systematic Errors," IEEE Trans. Geosci. Remote Sens., 48(2), pp. 716-726.

Hua Fenfen, 2014. Elevation Inversion through Multi-baseline InSAR.

Jordan, R. L., 1980. The Seasat-A synthetic aperture radar systems. IEEE Journal of Oceanic Eng., pp. 154-164.

Krieger, G., Zink, M., Bachmann, M., and et al., 2012. TanDEM-X: a radar interferometer with two formation-flying satellites. Acta Astronautica, 89(8), pp. 83-98.

Moreira, A., Fiedler, H., and Hajnsek, I., 2007. TanDEM-X: A Satellite Formation for High-Resolution SAR Interferometry. IEEE Trans. Geosci. Remote Sens., 45(11), pp. 3317-3341.

Massonnet, D., Rossi, M., Carmona, C., et al.,1993. The displacement field of the landers earthquake mapped by radar interferometry. Nature, 364, pp. 138-142.

Wang Qingsong, 2011. Research on High-efficiency and High-precision Processing Techniques of Spaceborne Interferometric Synthetic Aperture Radar.

WangYanping, 2003. Studies on Calibration Model and Algorithm for Airborne Interferometric SAR.

Wang Mengmeng, 2014. Research on Interferometric Parameters Joint Calibration of Airborne Double-antenna InSAR System.

WU Danqin, 2017. Modeling and Methodology for Airborne and Spaceborne InSAR Calibration.

Zhang Qingjun, 2017. System Design and Key Technologies of the GF-3 Satellite. Acta Geodaetica et Cartographica Sinica, 46(3), pp. 269-277.

Zwally, H. J., Schutz, B., Abdalati, W., and et al., 2002. ICESat's laser measurements of polar ice, atmosphere, ocean, and land. Journal of Geodynamics,34(3-4), pp. 405-445. 